\title{
Elimination of a Brain Tract Increases Errors in Pathfinding by Follower Growth Cones in the Zebrafish Embryo
}

\author{
Ajay B. Chitnis, ${ }^{*}$ and John Y. Kuwada, ${ }^{*+\ddagger}$ \\ *Neuroscience Program \\ tDepartment of Biology \\ ¥Institute of Gerontology \\ University of Michigan \\ Ann Arbor, Michigan 48109
}

\section{Summary}

The early zebrafish brain contains a simple axon scaffold of longitudinal tracts connected by commissures. Neurons in the nucleus of the posterior commissure (nuc PC) project growth cones along a specific route in this axonal scaffold, raising the possibility that specific axons in the early scaffold guide nuc PC growth cones. We tested this possibility by analyzing the behavior of nuc PC growth cones in embryos in which a portion of the scaffold, normally traversed by nuc PC growth cones, was surgically prevented from forming. Under these conditions nuc PC growth cones extended along both normal and aberrant pathways. This suggests that specific axons do provide guidance cues, since their removal leads to errors. However, these cues are not obligatory, since some growth cones still followed normal pathways.

\section{Introduction}

Neurons make appropriate connections in the embryo by extending growth cones along defined pathways to reach their targets. Various cues have been shown to guide growth cones along these pathways. The cues may affect all growth cones or may be specific for a subset of growth cones. They may be mechanical or chemical in nature, located on neuronal or nonneuronal substrates in the pathway, contact mediated or secreted by a distant source, and operate by either promoting or inhibiting growth cone extension along a particular route (Goodman et al., 1984; Dodd and Jessell, 1988; Lumsden, 1988; Raper et al., 1988). For the most part, these mechanisms of growth cone guidance have been elucidated in vitro or in embryos with experimentally accessible nervous systems, but not in the more complex vertebrate brain. Recently it was demonstrated that the brain of the early zebrafish embryo has a surprisingly simple organization (Chitnis and Kuwada, 1990; Wilson et al., 1990). It contains a small number of identifiable clusters of neurons, whose growth cones can be followed as they navigate within a well-defined region of the forebrain and midbrain.

At $28 \mathrm{hr}$ postfertilization (PF), the zebrafish brain contains a simple scaffold of axonal tracts made up of longitudinal tracts and commissures (Figure 1). The midbrain contains two longitudinal tracts; the dorsolateral tract of the postoptic commissure (TPOC), and the ventromedial medial longitudinal fasciculus (MLF).
The TPOC originates from neurons in the anterior diencephalon and breaks up into 2-4 parallel bundles in the anterior tegmentum. The MLF originates from neurons in the nucleus of the MLF, located in the anterior tegmentum and remains a tightly fasciculated tract throughout its extent. The two longitudinal tracts on the two sides of the brain are connected by the posterior commissure (PC). The PC is located at the junction of the forebrain and midbrain (Figure 1). The PC crosses the dorsal midline connecting the two sides of the anterior tegmentum. The axons of the TPOC, the MLF, and the ventral tegmental commissure (TC) also course through the anterior tegmentum. Additionally, the dorsoventral diencephalic tract (DVDT) intersects the TPOC in the posterior diencephalon approximately $60 \mu \mathrm{m}$ anterior to the PC.

Within this axonal scaffold, the neurons of the nucleus of the posterior commissure (nuc PC) project growth cones that extend along a stereotyped route (Chitnis and Kuwada, 1990). Early in development the nuc PC consists of 3-4 neurons and is located lateral to the dorsal midline and just posterior to the PC. We have investigated mechanisms that guide these growth cones by analyzing their outgrowth following alteration of the environment they normally traverse.

Results and Discussion

\section{Pathfinding by Nuc PC Growth Cones}

The growth cones of the nuc PC neurons project ventrally along the $\mathrm{PC}$ to reach the intersection of the TPOC, MLF, and TC in the anterior tegmentum by $22-$ $24 \mathrm{hr}$ PF ( $\mathbf{n}=9)$ ). Presumably, the growth cones of the nuc PC neurons could grow along any one of these tracts, but they always exit the PC by turning posteriorly along the TPOC toward the hindbrain without sending collaterals along the other tracts $(n=14$; Figure 2). It is unlikely that this behavior is due to nuc PC growth cones nonspecifically fasciculating with the first axons they encounter. If this were the case, the nuc PC growth cones would be expected to continue to extend ventrally on the $P C$ rather than turn onto the TPOC, since they are already following the PC.

The stereotyped extension of the nuc PC growth cones along the TPOC could be accounted for by various alternative hypotheses. Each hypothesis makes a different prediction about the behavior of the nuc PC growth cones if prevented from having access to the TPOC axons. According to one hypothesis, the growth cones may be capable of growing on the other tracts at the intersection, but cues associated with the TPOC axons may specifically promote the extension of nuc PC growth cones, resulting in growth along this pathway. This hypothesis predicts that in the absence of the TPOC axons, the nuc PC growth cones would grow aberrantly along other axonal tracts at the intersection. In an alternative hypothesis, the TPOC path- 


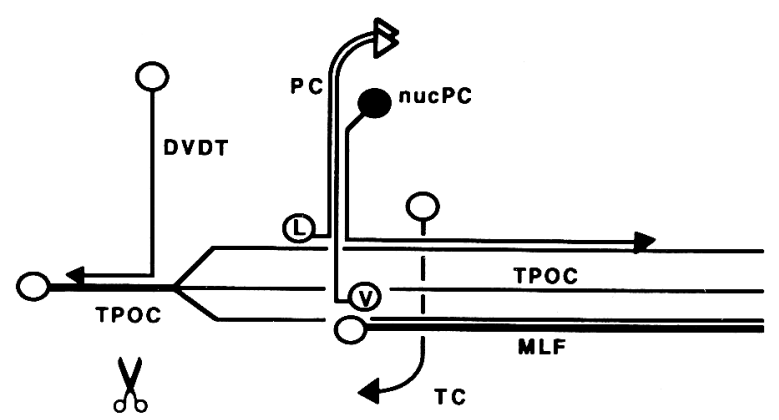

Figure 1. Schematic of the Early Axonal Scaffold Showing the Intersection of Tracts in the Anterior Tegmentum

Open circles represent clusters of neurons that establish the axonal scaffold. $L$ and $V$, the lateral and ventral clusters of neurons that contribute to the formation of the posterior commissure (PC). TPOC, tract of the postoptic commissure; DVDT, dorsoventral diencephalic tract; $M L F$, medial longitudinal fasciculus; TC, tegmental commissure; nucPC, nucleus of the posterior commissure. In this and all other figures unless noted otherwise, anterior is to the left and dorsal is up.

way may be taken by default. Physical barriers may prevent nuc PC growth cones from having access to the other pathways, or cues associated with the other tracts may inhibit the growth of nuc PC growth cones. This hypothesis predicts that in the absence of the TPOC axons, the nuc PC growth cones would not grow along the other axonal tracts at the intersection. A third possibility is that cues in the tegmentum along the pathway of the TPOC, independent of the TPOC axons themselves, may guide the nuc PC growth cones. This hypothesis predicts that in the absence of the TPOC axons, the nuc PC growth cones would continue to follow their stereotyped pathway. We have tested these hypotheses by analyzing the behavior of the nuc PC growth cones in embryos in which the growth cones do not have access to the TPOC axons.

\section{Nuc PC Growth Cones Follow Both Aberrant and Normal Pathways after Elimination of the TPOC}

The TPOC axons are projected early in development by neurons in the diencephalon, and the earliest ones normally reach the intersection at $20 \mathrm{hr}$ PF (Chitnis and Kuwada, 1990). These axons can be eliminated from the tegmentum by making a transverse cut through the posterior diencephalon at $16-20 \mathrm{hr}$ PF, before most of the TPOC axons have reached the midbrain. Electron micrographs of embryos operated in this manner between 19 and $20 \mathrm{hr}$ PF and assayed $8 \mathrm{hr}$ later showed no obvious axonal bundles in the region of the tegmentum where TPOC bundles are usually seen ( $n=2$; Figure 3). Furthermore, the effectiveness of the manipulation was tested by labeling axons with an antibody against acetylated tubulin (Piperno and Fuller, 1985; Chitnis and Kuwada, 1990; Figure 4A). With the exception of the TPOC, the scaffold of tracts, labeled with the acetylated tubulin antibody, was normal following this manipulation (Figures $4 \mathrm{~A}$ and $4 \mathrm{~B}$ ). Additionally, we confirmed that neurons located near the intersection in the tegmen-
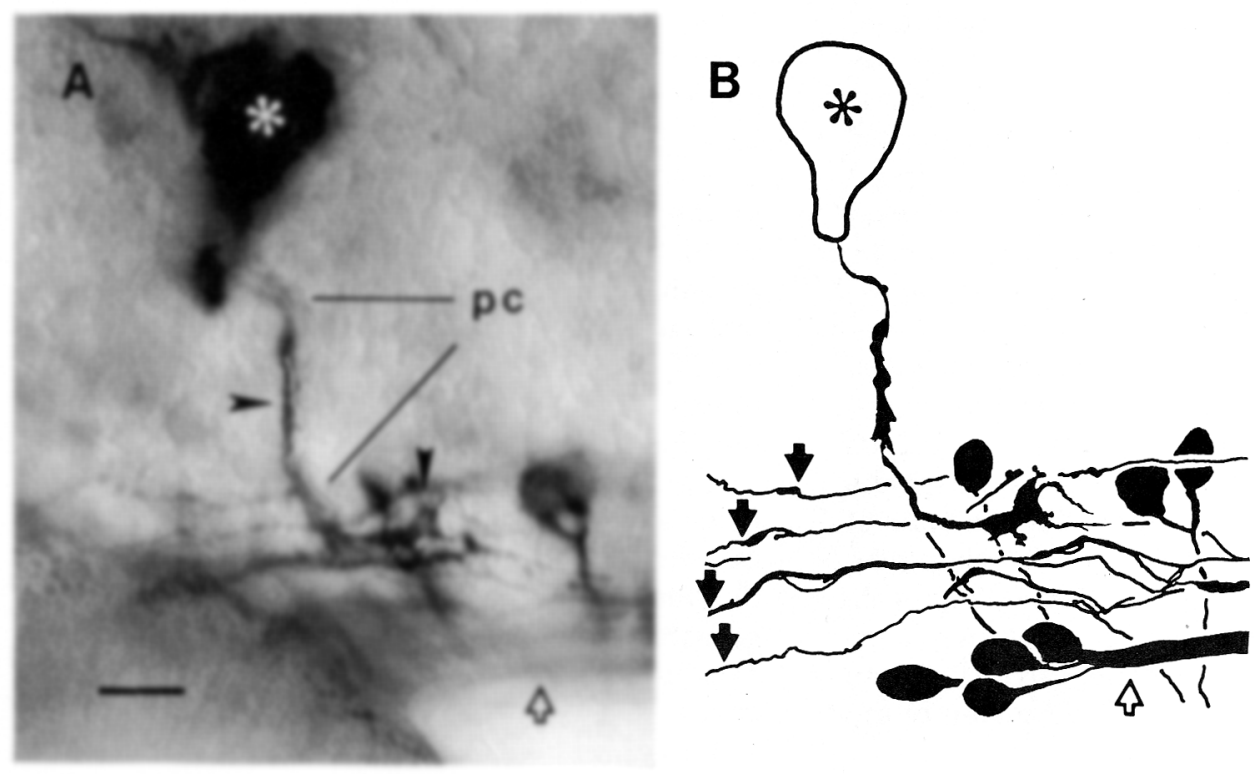

Figure 2. Nuc PC Growth Cones Follow PC Axons into the Intersection and TPOC Axons Out of the Intersection

(A) Micrograph showing 2 nuc PC growth cones (arrowheads), 1 extending ventrally on the PC and the other extending posteriorly on one of the TPOC axon bundles at $24 \mathrm{hr}$ PF. Asterisk, Dil application site; closed arrowheads, TPOC axons; open arrow, MLF. The nuC $\mathrm{PC}$ was inadvertently pulled anteriorly by the Dil electrode. In this embryo, the nuc PC growth cones were labeled brown and the other axons were labeled blue-violet (see Experimental Procedures). (B) Camera ludica drawing of the preparation shown in (A). Bar, $25 \mu \mathrm{m}$. 

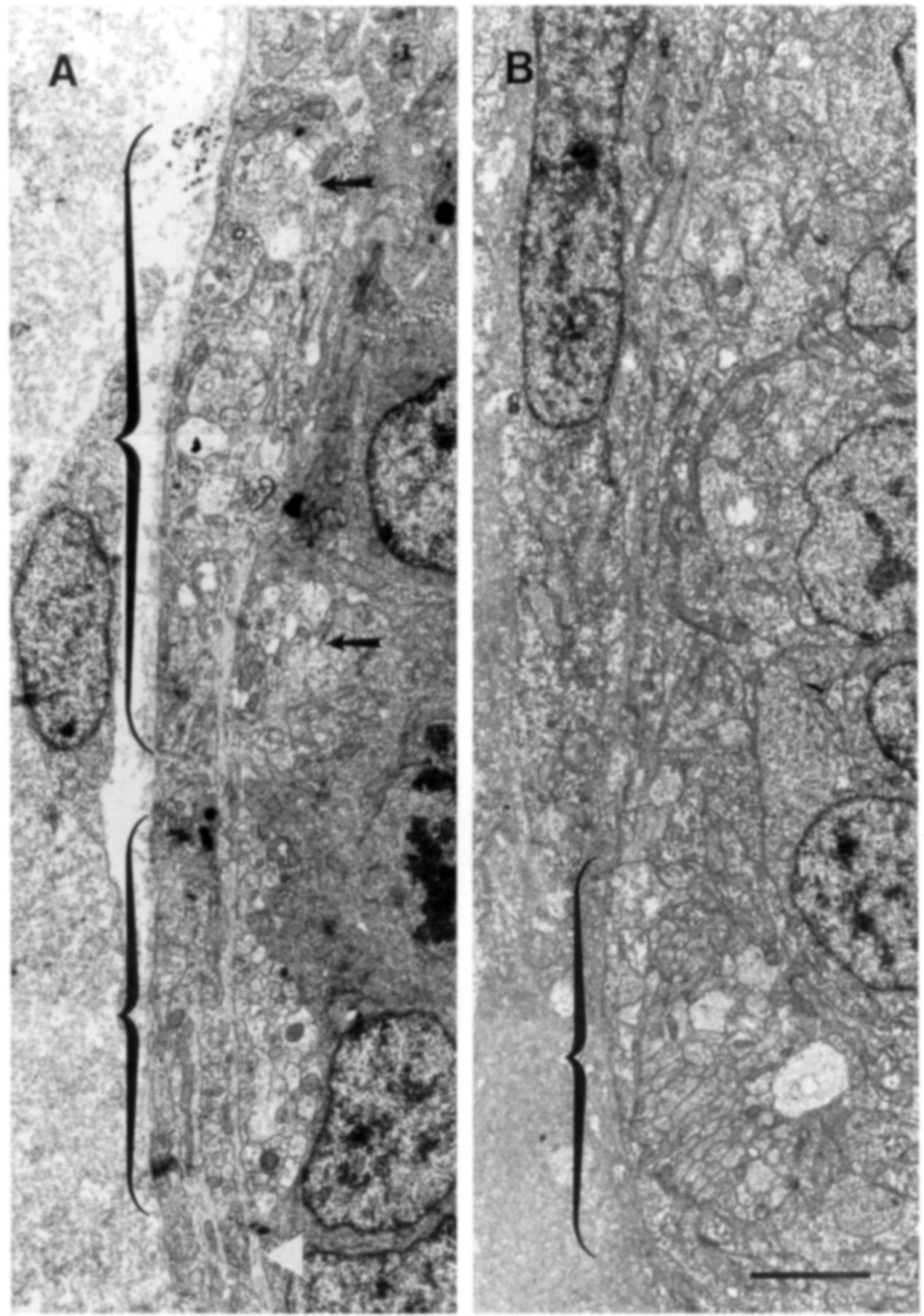

Figure 3. Elimination of the TPOC Axonal Bundles from the Tegmentum

Electron micrographs of transverse sections through the tegmentum at $28 \mathrm{hr}$ PF. Top is lateral and the bottom medial. (A) A normal embryo showing the MLF (smaller brackets) and TPOC (larger brackets). Some of the TPOC bundles are denoted by arrows, and some circumferential axons (white arrowhead) probably belonging to the tegmental commissural neurons are seen in this section. (B) An embryo in which a transverse cut was made in the caudal forebrain between 19 and $20 \mathrm{hr}$ PF. The MLF is present, but the TPOC axonal bundles are not. In this section no circumferential axons are evident, but they were present in other sections from this experimental embryo.

Bars, $3 \mu \mathrm{m}$. 


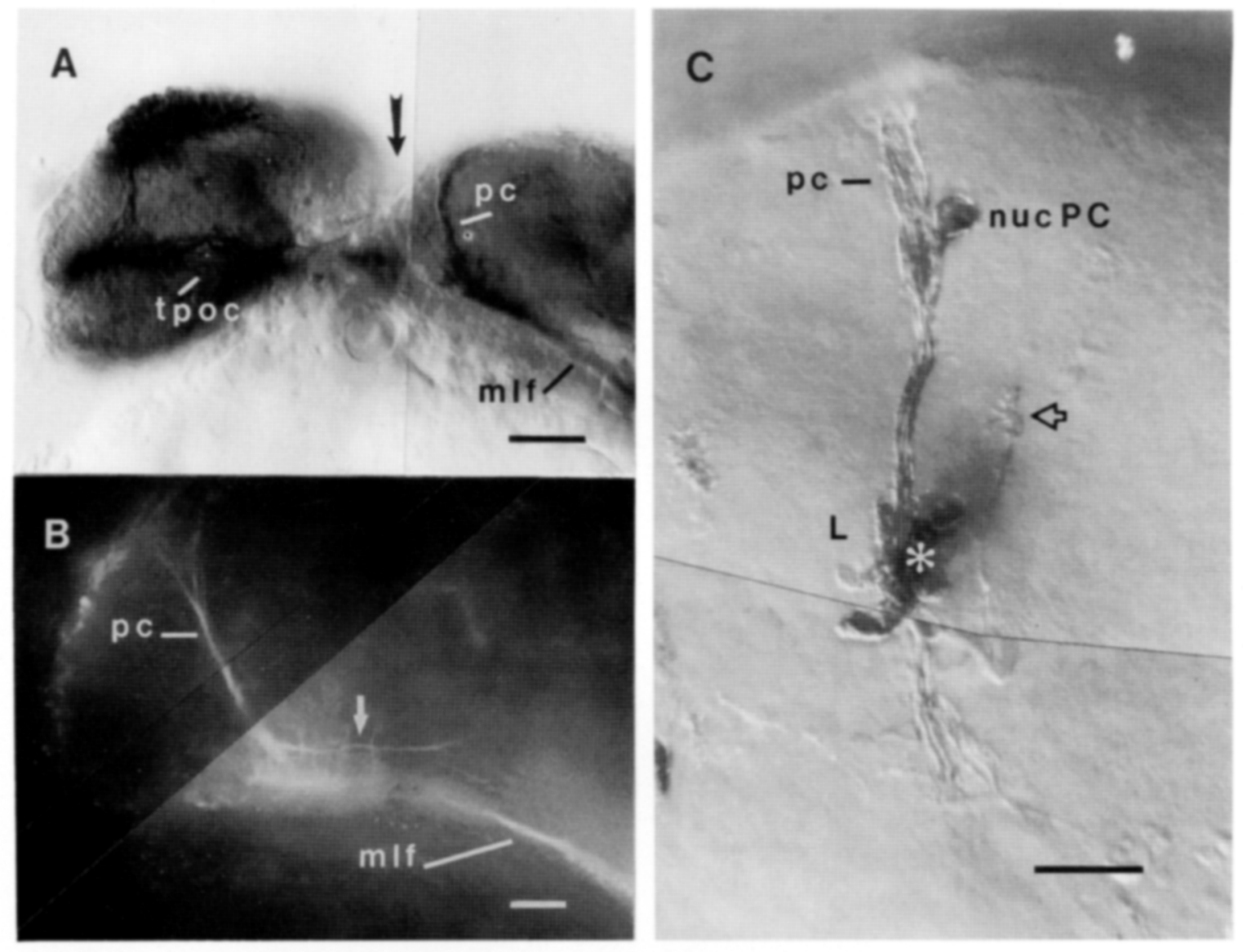

Figure 4. Development of Axonal Tracts following Elimination of TPOC Axons from the Tegmentum

(A) Acetylated tubulin antibody was used to label an embryo $8 \mathrm{hr}$ after the forebrain was cut (arrow). The axonal scaffold developed normally with the exception that TPOC axons did not grow into the tegmentum, nor did the nuc PC axons project along their normal pathway, since no axons were seen in the dorsolateral tegmentum.

(B) Midbrain of an embryo $8 \mathrm{hr}$ following the forebrain cut. No TPOC axons invaded the tegmentum, but axons that appeared to be from the $\mathrm{PC}$ were seen in the dorsolateral tegmentum (arrow). Axons were labeled by a diaminobenzidine reaction product (A) or a fluorescent marker (B) following application of the acetylated tubulin antibody.

(C) The lateral cluster of PC neurons (L) projected axons into the PC, as it normally does during the $8 \mathrm{hr}$ following the forebrain cut. The lateral cluster was labeled by injection of $\mathrm{Dil}$ (asterisk) into the cluster followed by photooxidation. In $4 / 8$ embryos the Dil retrogradely labeled a nuc PC neuron as well. In each of these embryos, an axon was seen following either the normal path of a nuc PC neuron or an aberrant path, as shown here (arrow). It is possible that these axons belonged to the back-labeled cell. Bars, $20 \mu \mathrm{m}$.

tum were normal. Three clusters of neurons, the ventral PC cluster, the lateral PC cluster, and the nuc PC, project axons into the PC (Chitnis and Kuwada, 1990; Figure 1). The neurons of the ventral and lateral PC clusters have axons that run dorsally and cross the dorsal midline in the PC; the cell bodies of the lateral cluster are located close to the intersection of tracts in the anterior tegmentum. Application of Dil to the cell bodies of the lateral cluster showed that these neurons had axons that projected normally in the PC following the manipulation ( $n=8$; Figure $4 \mathrm{C}$ ).

The TPOC failed to invade the tegmentum, as judged by labeling axons with the antibody to acetylated tubulin in 71 manipulated embryos. Of these, 26 embryos had no longitudinal axons in the dorsolateral tegmentum, the normal location of the TPOC, and the longitudinal portions of the nuc PC axons.
In the remaining 45 embryos longitudinal axons did occupy the dorsolateral tegmentum. These axons were not TPOC axons, since no axons were seen to cross the region that had been cut, and could have been nuc PC axons. However, their identity could not be unambiguously determined, since the acetylated tubulin antibody labels many if not all axons. There fore, the nuc PC axons were aberrant in at least 37\% of embryos and may have been normal in as many as $63 \%$ of embryos in the absence of the TPOC.

To identify the pathways taken by nuc PC growth cones in the absence of the TPOC, 64 nuc PC neurons were labeled with Dil or Lucifer yellow in an additional 39 embryos manipulated at 19-20 hr PF. Subsequently, the axonal tracts in these embryos were labeled with the acetylated tubulin antibody (Figure 5). In 16 of these embryos the manipulation failed to prevent the 

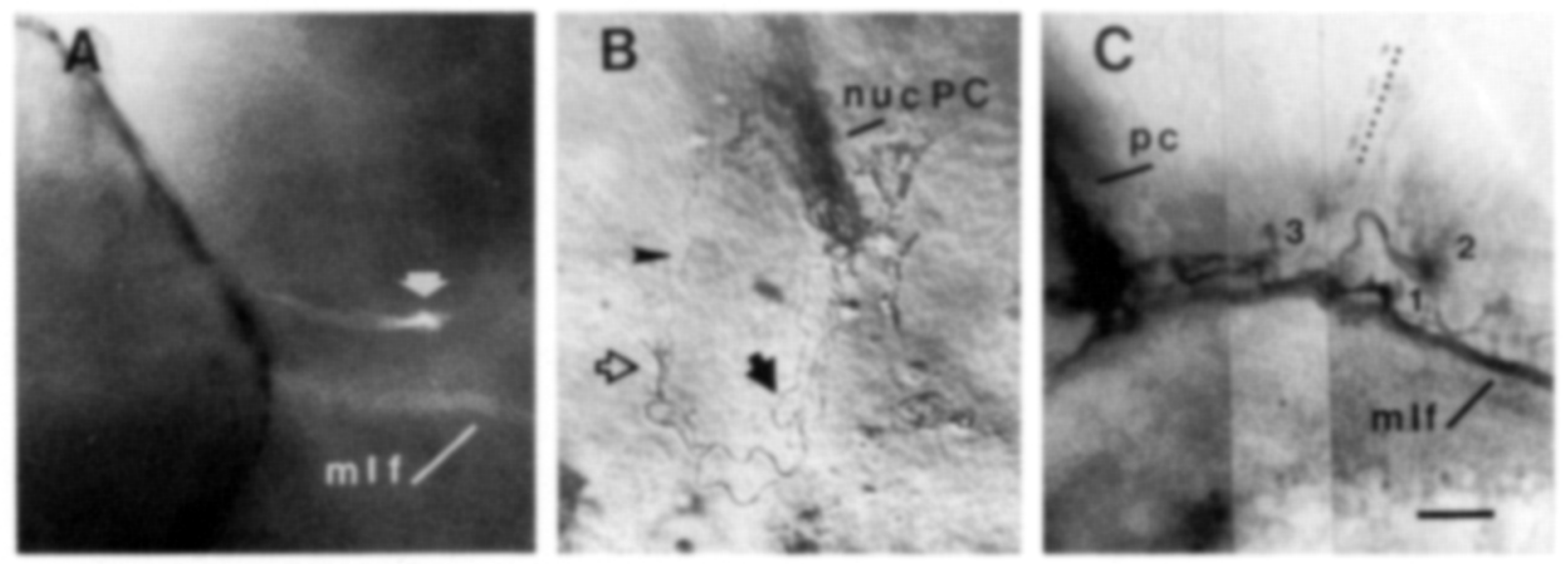

Figure 5. Nuc PC Growth Cones Follow Both Normal and Aberrant Pathways in the Absence of TPOC Axons

Nuc PC growth cones were labeled with Dil or Lucifer yellow, and other axons were labeled with the acetylated tubulin antibody. In each case shown, TPOC axons failed to invade the tegmentum.

(A) Nuc PC growth cone labeled with Lucifer yellow (arrow) following an apparently normal, dorsolateral pathway in the tegmentum in the absence of TPOC axons. The MLF labeled with the acetylated tubulin antibody and a TRITC-coupled secondary antibody can be seen ventral to the growth cone.

(B) A nuc PC growth cone labeled with Dil (open arrow) extended aberrantly up the DVDT (arrowhead). Pigment cells are seen to the right of the PC. Other nuc PC growth cones made a hairpin turn and grew aberrantly back up the PC (closed arrow). In this preparation, Dil-labeled axons were photooxidized and other axons were subsequently labeled with the acetylated tubulin antibody followed by a fluorescently labeled secondary antibody.

(C) Three nuc PC growth cones and axons labeled with Dil (1, 2, 3). All three axons ran along the dorsolateral tegmentum for some distance, but near the midbrain-hindbrain junction (dashed line), axons 1 and 2 merged aberrantly with the MLF for a short distance. Growth cones 2 and 3 were located in the dorsolateral midbrain-hindbrain, but growth cone 1 was ventromedial. In this preparation Dil-labeled cells were photooxidized and marked with the diaminobenzidine reaction product; other axons (including the MLF) were labeled with the acetylated tubulin antibody and visualized with the chloronaphtol reaction product. Bars, $20 \mu \mathrm{m}$.

TPOC from extending into the midbrain; these embryos served as controls. In these control embryos 25/ 27 labeled nuc PC growth cones extended along their stereotyped dorsolateral pathway along the TPOC axons (data not shown). The manipulation did successfully prevent the TPOC axons from extending into the tegmentum in the other 23 embryos. In these embryos, nuc PC neurons ( $n=37$ ) had axons that ran normally to the anterior tegmentum, but 17 of the neurons had growth cones that followed aberrant pathways once at the anterior tegmentum. Of these, 15 growth cones followed inappropriate axonal tracts (MLF, DVDT, and the PC) and 2 took pathways apparently devoid of other axons. Furthermore, 20/37 labeled growth cones extended along a dorsolateral longitudinal tegmental pathway, which corresponds to their normal route.

Our earlier study indicated that normally the earliest TPOC axon reaches the level of the anterior-most neurons in the nuc MLF (the location of the intersection) by $20 \mathrm{hr}$ PF (Chitnis and Kuwada, 1990). Since the manipulations were performed at $19-20 \mathrm{hr} P F$, it is possible that in some embryos the earliest TPOC axons had already reached the intersection at the time of the manipulations. Therefore, we cannot rule out the possibility that in those cases, cells and/or structures in the intersection were somehow modified by the TPOC growth cones to become effective cues for the nuc PC growth cones, or that the remnants of the
TPOC axons and growth cones persisted for $2-5 \mathrm{hr}$ to guide nuc PC growth cones. Pathfinding by the nuc PC growth cones would be normal in such embryos and abnormal in other embryos in which the TPOC growth cones never reached the anterior tegmentum. To test this possibility, the same manipulation was performed in 13 embryos at 16-17 hr PF and the embryos were allowed to develop for 11-12 hr. Nuc PC neurons were then assayed by intracellular injection of Lucifer yellow or orthograde labeling with Dil followed by labeling of all tracts with the acetylated tubulin antibody. The maturity of the tracts of 16-17 hr embryos was determined by labeling embryos from the same clutch used for the manipulations with the acetylated tubulin antibody. In every case the growth cones of the TPOC neurons were at least $40 \mu \mathrm{m}$ anterior to the intersection in these 16-17 hr embryos ( $n=11$; Figure $6 \mathrm{~A}$ ), in accordance with earlier results (Chitnis and Kuwada, 1990). As with the previous experiment, the TPOC failed to invade the tegmentum following a transverse cut through the diencephalon at 16-17 hr PF and nuc PC growth cones followed variable pathways, including their normal one (Figures $6 \mathrm{~B}$ and $6 \mathrm{C}) ; 6 / 22$ growth cones followed aberrant pathways, and 16/22 growth cones followed the normal longitudinal pathway in the dorsal tegmentum. These results suggest that it is not necessary for TPOC growth cones to ever reach the anterior tegmentum in order for nuc PC growth cones to follow their normal 

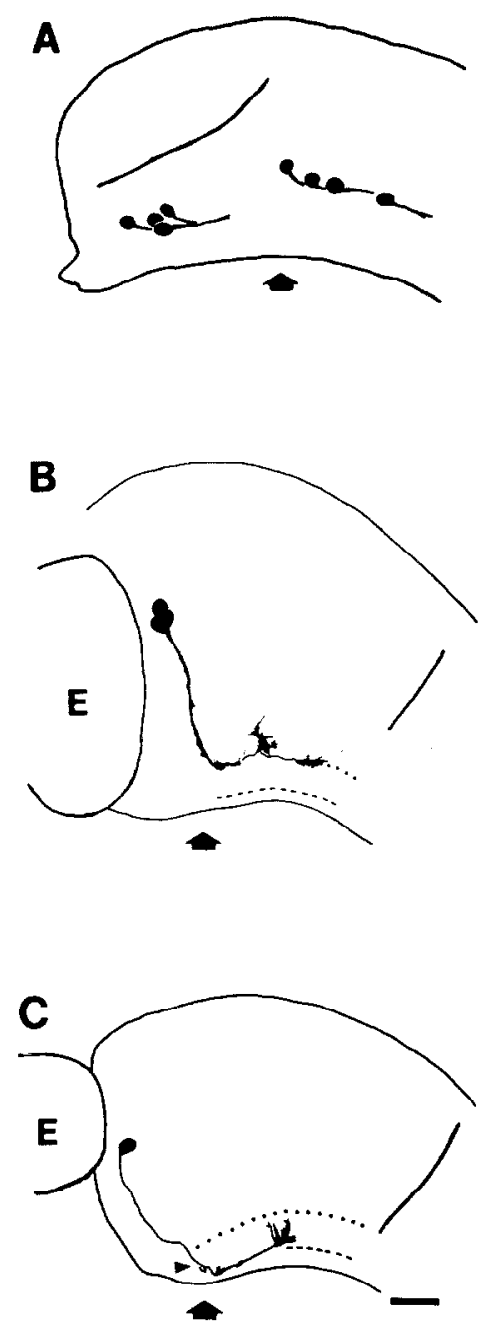

Figure 6. TPOC Growth Cones Need Not Ever Reach the Anterior Tegmentum for Nuc PC Growth Cones to Follow Their Normal Pathway

Cameral lucida drawings of side views of the brain with dorsal up and anterior to the left.

(A) A 17 hr embryo showing that the TPOC axons had not reached the anterior tegmentum. The axons in this embryo were labeled with an antibody to acetylated tubulin. Two clusters of neurons are shown. On the left are TPOC neurons; on the right, the neurons of the nucleus of the MLF. The PC has not yet formed, but its future position is denoted by the arrow.

(B) Trajectories taken by 3 nuc. PC growth cones in an embryo manipulated between 16 and $17 \mathrm{hr}$ PF and assayed $11 \mathrm{hr}$ later. One growth cone has taken its usual path in the dorsolateral tegmentum toward the midbrain-hindbrain border; a second growth cone has extended normally for a short distance and then aberrantly turned dorsal; a third growth cone has just made its correct turn in the anterior tegmentum. Nuc PC growth cones were labeled with Dil, and the other axons were labeled with the acetylated tubulin antibody. Dashed lines indicate the position of the MLF; dotted lines, the normal position of the missing TPOC; E, eye.

(C) A nuc PC growth cone aberrantly following the MLF in another experimental embryo. The axon looped (arrowhead) in the tract intersection of the anterior tegmentum.

Bar, $25 \mu \mathrm{m}$. pathway. Nuc PC neurons with aberrant trajectories had growth cones that apparently turned anterior at the tegmental intersection, extended along the MLF, or turned dorsal into the tectum after following their normal pathway in the dorsolateral tegmentum.

It is unlikely that the variable results of the manipulation on outgrowth by nuc PC axons are due to variability in the effectiveness of the manipulation to prevent TPOC axons from entering the tegmentum. There were no TPOC axons labeled by the acetylated tubulin antibody in the tegmentum following the manipulation, and a number of reasons indicate that this antibody is a reliable marker of axons in the zebrafish embryo. First, we had shown earlier that this antibody reliably labels all the axonal tracts in the early brain (Chitnis and Kuwada, 1990). Second, the antibody may label all axons within a tract, since at a particular stage electron micrographs of another tract, the DVDT (S. Wilson, personal communication), showed the same number of axons as in embryos with DVDT axons labeled by the acetylated tubulin antibody (Chitnis and Kuwada, 1990). Third, double labeling of the CoPA neuron, an identified class of commissural neuron in the spinal cord (Bemhardt et al., 1990), by intracellular injection of Lucifer yellow and application of the acetylated tubulin antibody showed that the neuron was labeled with the antibody up to the base of its growth cone at least as early as 1-2 hr after initial outgrowth from the cell body ( $n=4$; data not shown).

It is also not likely that variable, nonspecific effects of the manipulation can account for the aberrant pathways taken by all the nuc PC growth cones. First, the pattern of axons in the midbrain is normal with the exception that the TPOC axons are missing in the tegmentum. Second, the lateral cluster of PC neurons located near the intersection projected axons normally into the PC. This suggests that, with the exception of the TPOC, the region of the intersection was normal. Third, variability between embryos induced by the manipulations cannot account for the variable effects on the nuc PC growth cones. In 23 cases in which at least 2 nuc PC growth cones were labeled, all labeled growth cones followed normal pathways in 9 cases, all growth cones followed abnormal pathways in 4 cases, and growth cones followed both normal and abnormal pathways in $\mathbf{1 0}$ cases. The fact that nuc PC growth cones could follow different pathways from the same intersection in the same embryo also suggests that nuc PC growth cones need not follow each other.

It is possible that some, although not all, of the aberrant trajectories of nuc PC neurons are nonspecific outcomes attributable to damage, since the proportion of aberrant nuc PC trajectories was lower when the diencephalon was cut at $16-17 \mathrm{hr}$ compared with 19-20 hr PF. The additional $3 \mathrm{hr}$ following the manipulation at the earlier time may have allowed the embryo to recover from such damage. 


\section{Multiple Mechanism of Growth Cone Guidance}

Our results allow us to make a number of inferences about the nature of the cues that may be guiding the nuc PC growth cones. First, in the absence of the TPOC axons, a large proportion of the nuc PC growth cones make pathfinding errors in the anterior tegmentum. This signifies that cues associated with the TPOC axons normally play an important role in ensuring nearly error-free pathfinding by these growth cones. Second, the majority of nuc PC growth cones extended along an apparently normal, dorsolateral longitudinal pathway despite the absence of the TPOC axons. This suggests that cues found along this pathway in the tegmentum, independent of the TPOC axons themselves, may be capable of guiding the nuc PC growth cones. It is possible that these cues are the same ones that originally guide the TPOC growth cones. Previously, local cues associated with the diencephalon were shown to be important for guiding retinal axons in the amphibian embryo: retinal axons altered their trajectories when they encountered a rotated diencephalon graft (Harris, 1989). Our results are concordant with this observation and further suggest that both axonal and neuroepithelial cues may guide any given growth cone. Third, since most of the growth cones that extended along aberrant paths followed other axonal tracts, it is unlikely that physical barriers or inhibitory cues associated with these tracts normally prevent nuc PC growth cones from following these tracts.

Our findings are reminiscent of neuronal phenotypes in the cyc-1 and unc mutants of, respectively, zebrafish and nematodes. cyc-1 embryos are missing the floor plate cells, a specialized set of nonneuronal cells located at the ventral midline of the CNS (Hatta et al., 1991). Presumably as a consequence of this, a specific subset of spinal growth cones follow aberrant as well as apparently normal pathways at the ventral midline (Kuwada and Hatta, 1990, Soc. Neurosci., abstract). Likewise, mutations in the unc-5, unc-6, or unc- 40 genes also lead to a higher incidence of pathfinding errors by identified neurons, but again the same identified neurons often have normal trajectories in mutant nematodes (Hedgecock et al., 1990). One difference between our results and those in the nematode is that uniquely identified neurons varied in the nematode, whereas in our experiments neurons in a brain nucleus varied. Since we currently do not know whether all the nuc $P C$ neurons are identical, we cannot rule out the possibility that nuc PC neurons may be heterogeneous and that this may contribute to the variability in our results. However, since the behavior of the nuc PC growth cones is equivalent at the tegmental intersection (Chitnis and Kuwada, 1990), each nuc PC neuron would have to be utilizing a different set of guidance mechanisms at the intersection in order for heterogeneity among nuc PC neurons to account for our results.
Variable effects of manipulations on identified growth cones has also been reported in the grasshopper embryo. For example, application of antibodies against fasciclin II, an adhesion molecule found on a subset of axons in the CNS of embryonic grasshoppers (Bastiani et al., 1987; Grenningloh et al., unpublished data), caused the growth cones of a fasciclin IIpositive neuron either to extend along the correct pathway, albeit not as far as normal, or to bifurcate and extend a second growth cone along an aberrant pathway (Harrelson and Goodman, 1988).

Furthermore, in vitro and in vivo studies have demonstrated that multiple adhesion molecule systems may be active simultaneously during axonal outgrowth. In vitro studies on axonal outgrowth of chick ciliary ganglion neurons showed that simultaneous perturbation of two adhesion molecule systems ( $\beta 1$ integrin and $\mathrm{N}$-cadherin), by the application of antibodies to both classes of molecules, virtually eliminated neurite outgrowth; whereas antibodies to either class of molecules alone decreased outgrowth but did not eliminate it (Tomaselli et al., 1988). Neither a null mutation in the fas-1 gene nor a mutation in the $a b$ l gene results in gross morphological defects in the CNS of Drosophila embryos, but double mutants show clear defects in axonal outgrowth (Elkins et al., 1990).

In light of these findings, one interpretation of our results is that multiple mechanisms of growth cone guidance operate simultaneously to guide nuc PC growth cones in the brain. Elimination of the TPOC axons removes only one set of guidance cues. In the absence of the TPOC axons, nuc PC growth cones can still grow along their stereotyped pathway using other available cues in the tegmentum, but the probability of their choosing aberrant pathways is increased. Error-free navigation in the CNS may normally be brought about by the simultaneous operation of multiple guidance mechanisms, each of which is incapable of preventing errors on its own.

\section{Experimental Procedures}

Animals

Zebrafish embryos were collected from a laboratory breeding colony and maintained according to the procedures described in Myers et al. (1986).

\section{Dil Labeling}

Embryos were dissected to remove their yolk sacs, pinned in a Sylgard-lined embryo holder, fixed for 4-12 hr in 4\% paraformaldehyde in $0.1 \mathrm{M}$ phosphate buffer ( $\mathrm{pH} 7.4)$, washed, and placed on a fixed-stage Zeiss compound microscope outfitted with differential interference contrast optics and epifluorescence Growth cones were labeled orthogradely by pressure injection of small amounts of a $0.25 \%$ solution of Dil $(1,1$-dioctadecyl3,3,3,3-tetramethylindocarbocyanine perchlorate) in N,N-dimethylformamide (Honig and Hume, 1986) from a broken off microelectrode into the nuc PC or PC lateral cluster. Dil was allowed to spread for 4-12 hr, and the labeled neurons were marked with a brown reaction product by following the photooxidation 
procedure with diaminobenzidine and epifluorescence illumination (Maranto, 1982; Kuwada et al., 1990).

\section{Lucifer Yellow Injections}

Embryos were prepared as described above for Dil labeling and fixed for $6-8 \mathrm{~min}$ in $8 \%$ paraformaldehyde and $1 \%$ DMSO in $0.1 \mathrm{M}$ phosphate buffer. Nuc PC somata were visualized with differential interference contrast optics, impaled with microelectrodes filled with $10 \%$ Lucifer yellow in distilled water, and filled with dye by injecting hyperpolarizing currents $(0.1-0.5 \mathrm{nA}$ for $10-$ 120 s). Labeled neurons were viewed with epifluorescence and photographed and/or drawn with the aid of a camera lucida. In some cases Lucifer yellow-labeled neurons were also marked via the photooxidation procedure.

\section{Labeling of Axons with the Acelylated Tubulin Antibody}

Axons were labeled with the acetylated tubulin antibody (kindly provided by G. Piperno; Piperno and Fuller, 1985) using the whole-mount labeling procedure described by Patel et al. (1989). In many cases the axons of embryos with nuc PC growth cones labeled with Dil followed by photooxidation using diaminobenzidine as a chromagen were subsequently labeled by application of the acetylated tubulin antibody using chloronaphtol as a chromogen $(0.3 \%$ stock in methyl alcohol, diluted 1 to 5 with phosphate buffer), which gave a blue-violet reaction product. In some embryos a blue diaminobenzidine reaction product was produced by using a heavy metal intensification technique (Adams, 1981). In these embryos the nuc PC growth cones were brown, whereas all other axons were blue-violet. Similarly, the axons of some of the embryos in which nuc PC growth cones were labeled with Lucifer yellow were subsequently labeled with the acetylated tubulin antibody followed by a rhodamineconjugated secondary antibody.

\section{Elimination of the TPOC}

Embryos (16-17 hr and 19-20 hr PF) were stabilized in $2 \%-3 \%$ methyl cellulose in embryo-rearing solution (Kirchen and West, 1976), and the posterior portion of the forebrain was cut with fine scissors to prevent TPOC axons from projecting into the tegmentum. Care was taken not to damage the yolk sack during the cut. Operated embryos were washed with and incubaled in sterile Earle's balanced salt solution with an antibiotic/antimycotic cocktail (Sigma), in Hank's saline, or in fish Ringer's solution without glucose (Westerfield et al., 1986) for $8 \mathrm{hr}$.

\section{Electron Microscopy}

Embryos were processed for electron microscopy by fixing in $3 \%$ glutaraldehyde, $2 \%$ paraformaldehyde, $1 \%$ acrolein, $1 \%$ DMSO in $0.1 \mathrm{M}$ phosphate buffer for $1 \mathrm{hr}$; washed; postfixed in $2 \%$ osmium tetroxide in phosphate buffer for $1 \mathrm{hr}$; washed in $50 \mathrm{mM}$ sodium maleate buffer $(\mathrm{pH} 5.9)$; stained with $2 \%$ uranyl acetate in maleate buffer for 1-2 hr; washed in maleate buffer; dehydrated in an ethanol and propylene oxide series; embedded in plastic; and thin sectioned.

\section{Acknowledgments}

We thank J. Raper, K. Barald, H. Okamoto, and R. Bernhardt for helpful discussions; G. Piperno for the acetylated tubulin antibody; P. Knafl for technical assistance; and D. Bay for photography. This work was supported by grants from the National Institutes of Health (NS24848), the March of Dimes Birth Defects Foundation (5-687), and the Office of the Vice President for $\mathrm{Re}$ search at the University of Michigan. A. B. C. is supported by a Rackham Graduate Fellowship from the University of Michigan, and J. Y. K. is a Basil O'Connor Scholar of the March of Dimes Rirth Deferts Foundation.

The costs of publication of this article were defrayed in part by the payment of page charges. This article must therefore be hereby marked "advertisement" in accordance with 18 USC Section 1734 solely to indicate this fact.

Received December 28, 1990; revised May 6, 1991.

\section{References}

Adams, J. C. (1981). Heavy metal intensification of DAB-based HRP reaction product. J. Histochem. Cytochem. 29, 775.

Bastiani, M. J., Harrelson, A. L., Snow, P. M., and Goodman, C. S. (1987). Expression of fasciclin I and II glycoproteins on subsets of axon pathways during neuronal development in the grasshopper. Cell 48, 745-755.

Bernhardt, R. R., Chitnis, A. B., Lindamer, L., and Kuwada, J. Y. (1990). Identification of spinal cord neurons in embryonic and larval zebrafish. f. Comp. Neurol. 302, 607-616.

Chitnis, A. B., and Kuwada, J. Y. (1990). Axonogenesis in the brain of zebrafish embryos. J. Neurosci. 10, 1892-1905.

Dodd, J., and Jessell, T. M. (1988). Axon guidance and the patterning of neuronal projections in vertebrates. Science $242,692-$ 699.

Elkins, T., Zinn, K., McAllister, L., Hoffmann, F. M., and Goodman, C. S. (1990). Genetic analysis of a Drosophila neural cell adhesion molecule: interaction of fasciclin I and Abelson tyrosine kinase mutations. Cell 60, 565-575.

Goodman, C. S., Bastiani, M. J., Doe, C. Q., du Lac, S., Helfand, S. L., Kuwada, J. Y., and Thomas, J. B. (1984). Neuronal recognition during development: cellular and molecular approaches. Science $225,1271-1279$.

Harrelson, A. L., and Goodman, C. S. (1988). Growth cone guidance in insects: fasciclin $\|$ is a member of the immunoglobulin superfamily. Science 242, 700-708.

Harris, W.A. (1989). Local positional cues in the neuroepithelium guide retinal axons in embryonic Xenopus brain. Nature 399, 218-221.

Hatta, K., Kimmel, C. B., Ho, R. K., and Walker, C. (1991). The cyclops mutation blocks specification of the floor plate of the zebrafish central nervous system. Nature 350, 339-341.

Hedgecock, E. M., Culotti, ]. G., and Hall, D. H. (1990). The unc-5, unc- 6 , and unc- 40 genes guide circumferential migrations of pioneer axons and mesodermal cells on the epidermis in C. elegans. Neuron 4, 61-85.

Honig, M. G., and Hume, R. I. (1986). Fluorescent carbocyanine dyes allow living neurons of identified origin to be studied in long-term cultures. J. Cell Biol. 103, 171-187.

Kirchen, R. V., and West, W. R. (1976). The Japanese Medaka, Its Care and Development (Burlington, North Carolina: Carolina Biological Supply Co.).

Kuwada, J. Y., Bernhardt, R. R., and Nguyen, N. (1990). Development of spinal neurons and tracts in the zebrafish embryo. J. Comp. Neurol. 302, 617-628.

Lumsden, A. G. S. (1988). In The Making of the Nervous System, J. G. Parnvelas, C. D. Stern, and R. V. Stirling, eds. (Oxford: Oxford University Press), pp. 166-187.

Maranto, A. R. (1982). Neuronal mapping: a photooxidation reaction makes Lucifer yellow useful for electron microscopy. Science $217,953-955$

Myers, P.Z., Eisen, J. S., and Westerfield, M. (1986). Development and axonal outgrowth of identified motoneurons in the cebrafish. J. Neurosci. 6, 2278-2289.

Patel, N. H., Martin, B. E., Coleman, K. G., Poole, S. L., Ellis, M. C., Kornberg, T. B, and Goodman, C. S. (1989). Expression of engrailed proteins in arthropods, annelids, and chordates. Cell $58,955-968$.

Piperno, G., and Fuller, M.T. (1985). Monoclonal antibodies specific for an acetylated form of alpha-tubulin recognize the antigen in cilia and flagella from a variety of organisms. J. Cell Biol. 101, 2085-2094.

Raper, J. A., Chang, S., Kapthammer, I. P., and Rathjen, F. $G$ (1988). In The Making of the Nervous System, J. G. Parnvelas C. D. Stern, and R. V. Sterling, eds. (Oxford: Oxford University Press), pp. 188-203. 
Tomaselli, K. J., Neugebauer, K. M., Bixby, J. L., Lilien, L., and Reichardt, L. F. (1988). N-cadherin and integrins: two receptor systems that mediate neuronal process outgrowth on astrocyte surfaces. Neuron 1, 33-43.

Westerfield, M., McMurray, J.V., and Eisen, J. S. (1986). Identified motor neurons and their innervation of axial muscles in the zebrafish. J. Neurosci. 6, 2267-2277.

Wilson, S. W., Ross, L. S. Parrett, T., and Easter, S. J. (1990). The development of a simple scaffold of axon tracts in the brain of the embryonic zebrafish, Brachydanio rerio. Development 108, $121-145$. 